\title{
Biofilm Formation, Maturation and Prevention: A Review
}

\author{
Liaqat I'*, Liaqat $\mathrm{M}^{2}$, Ali $\mathrm{S}^{1}$, Ali NM³ , Haneef $\mathrm{U}^{4}$, \\ Mirza $\mathrm{SA}^{4}$ and Tahir $\mathrm{HM}^{1}$ \\ ${ }^{1}$ Department of Zoology, GC University, Lahore, Pakistan \\ ${ }^{2}$ Department of Nursing, Allama Iqbal Medical College, \\ Lahore \\ ${ }^{3}$ Govt. College for Women, Model Town, Lahore \\ ${ }^{4}$ Department of Botany, GC University, Lahore, Pakistan \\ *Correspondling author: Iram Liaqat, Department of \\ Zoology, GC University, Lahore, Pakistan
}

Received: December 11, 2018; Accepted: January 08, 2019; Published: January 15, 2019

\begin{abstract}
Biofilms are groups of microrganisms in which cells embedded in extracellular polysaccharide matrix (EPS) stick to each other on a surface. Bacterial polysaccharide is the major component of biofilm matrix and make it stable as well as resistant to disinfectants, biocides and antibiotics. Biofilms can be harmful or beneficial, however unfortunately negative effects outweighs the positive ones. Formation of biofilm may take place on both biotic and abiotic surfaces and may be monospecies or multispecies. Though multispecies biofilm are more prevalent in nature augmenting the need to communicate between inter and intra species. In fact, biofilm formation and quorum sensing (QS) are interconnected. Quorum sensing (QS) is a regulatory system that allows bacteria to produce and detect signal molecules and thereby communicate their behaviour in a cell-density dependent manner. Three main QS systems include: The acylhomoserine lactone (AHL) QS system in Gram-negative bacteria, the autoinducing peptide (AIP) QS system in Gram-positive bacteria and the autoinducer-2 (AI-2) QS system in both Gram-negative and -positive bacteria. Biofilm formation and associated problems are a serious emerging issue in household, industry and medical settings. Many antibiofilm strategies were suggested to cope with its formation rather than removal. Among these, four commonly include are: (1) use of broad spectrum antibiotics/biocides, (2) interference with bacterial EPS production, (3) quorum sensing (QS), and (4) flagellar assembly. A comprehensive knowledge of bacterial biofilm will help to develop effective control measures to prevent its formation at initial stages and to manage associated problems later, once developed and got mature completely.
\end{abstract}

Keywords: Biofilms; Autoaggregation; Prevention Strategies; Quorum Sensing; Bacterial Exopolymeric Compounds

\section{Introduction}

For more than 10 decades, microbiologists have studied homogeneous culture format of bacteria leading to tremendously informative physiology and genetics but biased picture of microbial life. Since the last 10 years, the concept of biofilms has gained enough significance and popularity. The fact about $99 \%$ of bacterial population exhibit biofilm mode at some stage in life has nourished maximally to this area of research. Bacterial aggregation happens due to numerous advantageous reasons including survival in extremely dynamic habitats sometimes with nutrient depletion, resistance to broad spectrum antibiotics, disinfectants and various phenotypic variations [1].

Biofilm is a structured consortium attached to a living or inert surface formed by microorganism adhering to each other and selfproduced extracellular polymeric substance (EPS). Depending upon species type, it comprise of 10-25\% cells and 75-90\% extracellular polymeric substances (EPS) matrix [2]. This indicates that EPS is much more than just a sticky substance that holds cells together. Indeed, the matrix imbues the biofilm with a range of attributes, including antibiotic resistance, storage of extracellular enzymes, nutrient capture, gradient formation and protection from stress that single cells or planktonic cells cannot achieve in its absence. In this way, the matrix is truly responsible for the emergent properties of the biofilm [3].
Biofilm research has become a revolutionized field and evolved tremendously with the advancement in computational sciences, various molecular techniques and omics' based technologies. Despite the fact, that monoculture of bacteria is easy to study and manipulate, it's also undeniable to accept that none of the habitat is occupied by just single bacterial species. There is always interaction and communication between different species microrganisms in any locality to make a stable community. Hence research in this emerging field of biofilm has led to development of new techniques and additional models [4], to understand its structure and mechanism. Researchers have answered questions for example, Multispecies biofilm is more stable and require less nutrients compared to monospecies biofilm $[5,6]$. It has increased tolerance to disinfectants, antimicrobials and predation [7]. Likewise, autotroph-heterotroph interactions, interaction, competition and cooperation between diverse microbial species using chemical signals [8], are all newly investigated areas of research.

Biofilm may be formed by a single bacterial species that makes it a monoculture also called monofilms or diverse species of bacteria may be involved in its structure, hence called as multiculture (also multifilms). Both types act as biocatalysts during different processes to transform organic substances. Monofilms are important to control the processes required for increased yield and quality of a desired product, whereas multispecies are valuable for multistage processes of biogas production and pharmaceutical formulations [9]. Quorum 


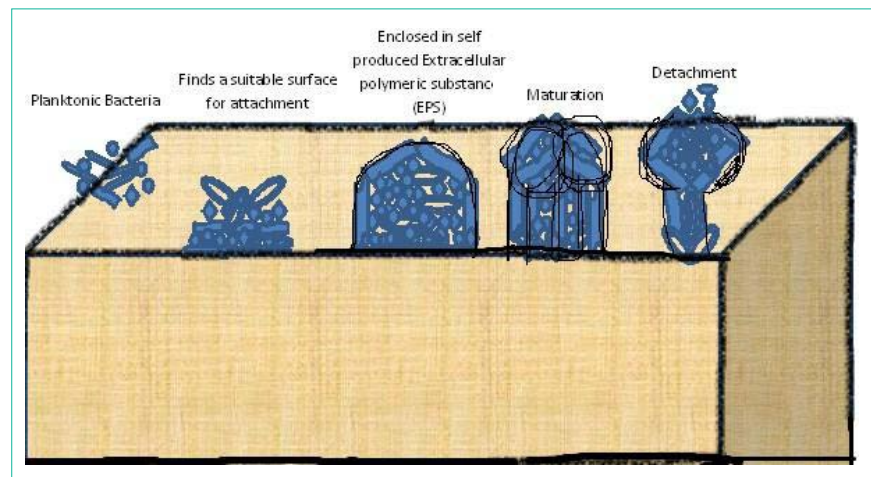

Figure 1: Formation of biofilm in four steps of adhesion, accumulation, maturation and detachment.

sensing (QS) plays key role to control mono and multispecies biofilm formation and other social/physiological behaviours including symbiosis, formation of spore or fruiting bodies, bacteriocin production, genetic competence, programmed cell death, virulence [10]. This process of intercellular communication, called quorum sensing, was first described in the marine bioluminescent bacterium Vibrio fischeri. In this system, bacteria in a community convey their presence by producing, detecting, and responding to small diffusible signal molecules called autoinducers. Bacterial QS system has generally been divided into three types: (1) Gram-negative bacteria use acyl-homoserine lactones (AHL) as signal molecules- LuxI/LuxRtype quorum sensing; (2) Gram-positive bacteria use small peptides as signal molecules called as oligopeptide-two-component-type QS (3) Both Gram-negative and Gram-positive bacteria has common luxSencoded autoinducer 2 (AI-2) QS. Each type of signalling system is detected and responded by proper sensing apparatus and regulatory control [11].

The implementation of any process of biofilms from biotechnology perspective require complete information of microrganisms involved in its formation. In general, it is a multistep process. First step is the process of bacterial attachment to either biotic or abiotic surfaces. Afterwards the attached cells mature and are enclosed in self produced extracellular polymeric substance (EPS), which is formed by a combination of proteins, polysaccharides and other biomolecules. It is responsible for three dimensional architectural form and stabilization of biofilms. It may be linear or branched molecules formed by one repeated sugar (homopolysaccharides) or by a mixture of different sugars (heteropolysaccharides). In final step, planktonic cells detach from biofilm matrix and start process again at new process (Figure 1) [12]. It is believed that environmental signals, flagella, outer membrane proteins, pili, or LPSs collectively led to the formation of microcolonies which becomes mature biofilm using QS molecules [13]. However, scientists are still looking for answers of questions concerning mechanisms involved in bacterial sensing of surface and development of different stages during biofilm development [14].

Surface sensing is very important phenomenon in various bacterial species. Various proteinaceous appendages including pili, fimbriae, flagella and outer membrane proteins play vital role in bacterial sensing. These are important for bacterial aggregation, bacterial host relationship, host colonization and motility [15].
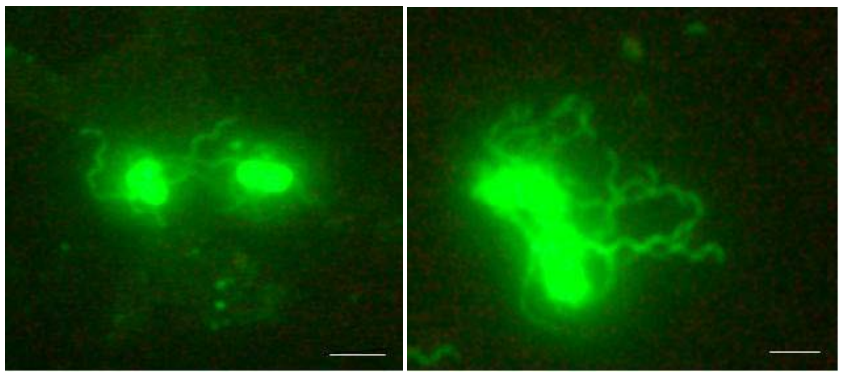

Figure 2: Phase contrast microscopy of Alexa Flour-488 labelled flagellar strains of Salmonella typhimurium. $=100 X$.

Initially, most of study to elaborate this was performed using marine bacterial species such as Vibrio parahaemolyticus. This rod shaped bacterium when occupy a surface, it exhibits unique phenotypic transformations. For example, in planktonic mode, it has just one polar flagellum, however, upon attachment to a surface it exhibits many lateral flagella thus facilitating its swarming motility. It was suggested that this bacterial adaptation was due to loss of rotation of its polar flagellum probably due to attachment to a surface. This fact was supported by the experimental data in which flagellar rotation was blocked by treating with a drug phenamil, that disrupts ion transport in flagellar basal body thus inhibit flagellar rotation and appearance of lateral flagella without sensing a surface [16]. Likewise, similar phenomenon was well explained in Pseudomonas aeruginosa providing novel insights into biofilm development.

Development of biofilm has been observed in various sectors of medical, industrial and domestic importance. Blockage of pipes, infections, corrosion, food spoilage, antibiotics failure, reduced efficacy of disinfectants and pesticides were all found to be biofilm's borne problems, we are facing currently and since more than a decade. More seriously, many human and animal disease outbreaks have been associated with biofilms and $80 \%$ of infections have been estimated to be linked with microbial biofilms. This explains that why there has been a lot of research in this area and methods/techniques to develop (1) resistant antibiotics/biocides as well as interfere with (2) bacterial EPS production, (3) quorum sensing (QS), and (4) flagellar assembly have been explored as possible strategies to either inhibit or interfere with this matrix enclosed bacterial growth.

The effective use of biocides and antibiotics is a basic challenge to target biofilm bacteria in industrial and health care sector due to increased resistance since these prefer to live as biofilm rather than planktonic. Biocide effectiveness in biofilms is compromised and $3 \mathrm{x}$ less compared to bacterial suspensions. However, this might not be correct due to heterogeneous nature of different parts of the biofilm having different susceptibilities for biocides. The rate of diffusion of any biocide or antibiotic is variable across a biofilm surface depending upon attached cells temperature, molecular size, and concentration gradient of the diffusing molecule [17]. Additionally, oxidative biocides are more preferred due to broad spectrum activity and multiple target sites against a diverse range of organic compounds. These biocides combined with surfactants are mostly utilized for disinfection and sanitation purposes in a variety of fields, such as hospitals, food manufacturing, and pharmaceutical industry [18]. 
Secondly, most of research on biofilms has seen EPS as a problem that requires it to be eliminated due to possible toxic effects for humans, animals and environment. It acts as glue and promote autoaggregation by establishing connection between cells, environments and signalling molecules. A mutant strain unable to synthesize EPS II (galactoglucan, from the symbiotic rhizobacterium Ensifer meliloti) will be unable to show an aggregative phenotype. Aggregation of the non-EPS-producing strains was observed by resuspending the cells in culture medium containing EPS II [19]. Aggregative behaviour in Pseudomonas aeruginosa is triggered by the presence of the toxic detergent SDS. This behaviour depends on production of the EPS PsI. Inactivation of either the psi gene or the c-di-GMP-mediated signaling system that activates the gene results in reduced autoaggregation [20].

It is well known that quorum sensing-mediated social activities favor microbial interactions and are believed as major mechanisms to regulate population-level virulence of bacteria. Additionally, growing evidence of bacterial quorum sensing systems in regulating cross-kingdom signaling with eukaryotes has led to develop QS interference as prime area to control biofilm formation and associated infections. Presently, four suggested ways to interfere with bacterial QS are (1) inhibiting signal generation; (2) interference with signal dissemination; (3) blocking of signal receptors; and (4) inhibiting signaling response system [21]. Evidences have shown that anti QS compounds derived from both plants and algae exist in nature. One major source is the red seaweed called Delisea pulchra (Greville) that grows under the sea around Australia, produces a range of biologically active furanones. These compounds blocks signal molecules or their interactions with receptors hence inhibit bacterial quorum sensing along with associated virulent activities. These prevent bacterial colonization by interfering with acyl-HSL signaling systems [22], AI-2 signaling systems in both Gram-negative and -positive bacteria $[23,24]$, expression of bacterial exo-enzymes that degrade components of the host immune system [21]. These signal analogs specifically block or override the bacterial command line and control the harmful activities of bacteria without inhibiting their growth. These differ from biocides or antibiotics in a way that these put no selection pressure on bacteria hence preventing resistant microbes [25].

Flagella-mediated motility plays important role in different stages during biofilm formation. These enable the free-swimming phenotype to reach a suitable surface and the surface-motile phenotype to adhere to, develop into mature form and finally dissociate to move on another surface (Figure 2). Motility-defective mutants of $P$. aeruginos $a$ and $E$. coli were unable to attach to surfaces or to develop a mature biofilm $[26,27]$. These appendages thus have a dual role as motile machines and adhesions that move and fix bacteria to surfaces and among surfaces. The complex association between motility and biofilm formation involves the use of a particular structure for different functions at different stages and requires the precise integration of environmental and cellular signals [28].

\section{Conclusion}

Biofilms represent a threat for human health and to industry. The approaches capable of preventing biofilm are more advantageous instead of treating biofilm related problems. Though there are shortcomings in different strategies, however improvement or modulation of existing technology to prevent biofilm formation remains the most effective and promising way to deal with associated problems in different sectors. Multidisciplinary studies using new approaches are suggested to understand how bacteria move, attach and interact in different environments. Such detailed information will enhance our knowledge of biofilm formation and its interaction with eukaryotes or even other higher organisms.

\section{References}

1. Nadell CD, Xavier JB, Foster KR. The sociobiology of biofilms. FEMS Microbiol Rev. 2009; 33: 206-224.

2. Flemming HC, Wingender J. The biofilm matrix. Nat Rev Microbiol. 2010; 8: 623-633.

3. Flemming HC, Wingender J, Szewzyk U, Steinberg P, Rice SA, Kjelleberg S. Biofilms: An emergent form of bacterial life. Nat Rev Microbiol. 2016; 14: 563-575.

4. Cole JK, Hutchison JR, Renslow RS, Kim YM, Chrisler WB, Engelmann HE, et al. Phototrophic biofilm assembly in microbial-mat-derived unicyanobacterial consortia: Model systems for the study of autotroph-heterotroph interactions. Front Microbiol. 2014; 5: 109.

5. Burmolle M, Webb JS, Rao D, Hansen LH, Sorensen SJ, and Kjelleberg S. Enhanced biofilm formation and increased resistance to antimicrobial agents and bacterial invasion are caused by synergistic interactions in multispecies biofilms. Appl Environ Microbiol. 2006; 72: 3916-3923.

6. Ren D, Madsen JS, Sorensen SJ, Burmolle M. High prevalence of biofilm synergy among bacterial soil isolates in cocultures indicates bacterial interspecific cooperation. ISME J. 2015; 9: 81-89.

7. Lee KWK, Periasamy S, Mukherjee M, Kjelleberg S, Rice SA. Biofilm development and enhanced stress resistance of a model, mixed species community biofilm. ISME J. 2014; 8: 894-907.

8. Fiegna F, Moreno-Letelier A, Bell T, Barraclough TG. Evolution of species interactions determines microbial community productivity in newenvironments. ISME J. 2015; 9: 1235-1245.

9. Maksimova YuG. Microbial biofilms in biotechnological processes. Appl Biochem Microbiol. 2014; 50: 750-760.

10. Parsek MR, Greenberg EP. Sociomicrobiology: The connections between quorum sensing and biofilms. Trends Microbiol. 2005; 13: 27-33.

11. Federle MJ, Bassler BL. Interspecies communication in bacteria. J Clin Invest. 2003; 112: 1291-1299.

12. Kim SJ, Chang J, Rimal B, Yang H, Schaefer J. Surface proteins and the formation of biofilms by Staphylococcus aureus. Biochim Biophys Acta Biomembr. 2018; 1860: 749-756.

13. Rinaudi LV, Giordano W. An integrated view of biofilm formation in rhizobia. FEMS Microbiol Lett. 2010; 304: 1-11.

14. Armbruster CR, Parsek MR. New insight into the early stages of biofilm formation. Proc Natl Acad Sci USA. 2018; 115: 4317-4319.

15. Mora P, Rosconi F, Franco Fraguas L, Castro-Sowinski S. Azospirillum brasilense $\mathrm{Sp} 7$ produces an outer-membrane lectin that specifically binds to surface-exposed extracellular polysaccharide produced by the bacterium. Arch Microbiol. 2008; 189: 519-524.

16. McCarter L, Silverman M. Surface-induced swarmer cell differentiation of Vibrio parahaemolyticus. Mol Microbiol. 1990; 4: 1057-1062.

17. De Kee D, Liu Q, Hinestroza J. Viscoelastic (non-fickian) diffusion. Can J Chem Eng. 2005; 83: 913-929.

18. Liaqat I, Sabri AN. Effect of biocides on biofilm bacteria from dental unit water lines. Curr Microbiol. 2008; 56: 619-624.

19. Sorroche FG, Rinaudi LV, Zorreguieta A, Giordano W. EPS II-dependent autoaggregation of Sinorhizobium meliloti planktonic cells. Curr Microbiol. 2010; 61: 465-470. 
20. Klebensberger J, Birkenmaier A, Geffers R, Kjelleberg S, Philipp B. SiaA and $\mathrm{SiaD}$ are essential for inducing autoaggregation as a specific response to detergent stress in Pseudomonas aeruginosa. Environ Microbiol. 2009; 11 3073-3086.

21. Zhang LH, Dong YH. Quorum sensing and signal interference: Diverse implications. Mol Microbiol. 2004; 53: 1563-1571.

22. Hentzer M, Givskov M. Pharmacological inhibition of quorum sensing for the treatment of chronic bacterial infections. J Clin Invest. 2003; 112: 1300-1307.

23. Liaqat I, Bachmann RT, Sabri AN, Edyvean RG, Biggs CA. Investigating the effect of patulin, penicillic acid and EDTA on biofilm formation of isolates from dental unit water lines. Appl Microbiol Biotechnol. 2008; 81: 349-358.

24. Liaqat I, Bachmann RT, Sabri AN, Edyvean RG. Isolate-specific effects of patulin, penicillic Acid and EDTA on biofilm formation and growth of dental unit water line biofilm isolates. Curr Microbiol. 2010; 61: 148-156.
25. Liaqat I, Bachmann RT, Edyvean RG. Type 2 quorum sensing monitoring, inhibition and biofilm formation in marine microrganisms. Curr Microbiol. 2014; 68: 342-351.

26. Liaqat I, Mirza S A, Iqbal R., Saleem G, Ali N M., Majid S. Flagellar Motility plays important role in biofilm formation of Bacillus cereus and Yersinia enterocolitica. Pak J Pharm Sci. 2018; 31: 2047-2052.

27. Kumar A, Ting YP. Presence of Pseudomonas aeruginosa influences biofilm formation and surface protein expression of Staphylococcus aureus. Environ Microbiol. 2015; 17: 4459-4468.

28. Verstraeten N, Braeken K, Debkumari B, Fauvart M, Fransaer J, Vermant $\mathrm{J}$, Michiels J. Living on a surface: Swarming and biofilm formation. Trends Microbiol. 2008; 16: 496-506.
J Bacteriol Mycol - Volume 6 Issue 1 - 2019

ISSN : 2471-0172 | www.austinpublishinggroup.com

Liaqat et al. () All rights are reserved
Citation: Liaqat I, Liaqat M, Ali S, Ali NM, Haneef U, Mirza SA, et al. Biofilm Formation, Maturation and Prevention: A Review. J Bacteriol Mycol. 2019; 6(1): 1092. 